\title{
Non-Overlapping DDMs to Solve Flow in Heterogeneous Porous Media
}

\author{
Dan-Gabriel Calugaru and Damien Tromeur-Dervout \\ University Lyon 1, MCS/CDCSP - ISTIL, 69622 Villeurbanne \\ (\{calugaru, dtromeur\}@cdcsp.univ-lyon1.fr)
}

Summary. For flow problems in multi-layered porous media, one can define a natural non-overlapping domain decomposition (DD). The simplest way to obtain DDMs is to distribute interface conditions (pressure and flux continuity) for each pair of adjacent subdomains and to use the Dirichlet-Neumann (D-N) algorithm. A different way is the use of two Robin conditions (RC) also distributed for each subdomain (Robin-Method). The main inconvenience of both methods is that the convergence is not ensured. To obtain efficient methods, we retain from previous works two basic ideas: an acceleration of Aitken type for the D-N algorithm and finding optimized coefficients for the Robin-Method. In the present paper, we analyze these improved algorithms in 1-D and 2-D framework for flow problems in heterogeneous porous media and we present a numerical comparison.

\section{Introduction}

Flow in heterogeneous porous media is to be solved in many hydrological or engineering applications, as oil recovery (Faille et al. [2001]), earthquake prediction (Calugaru et al. [2002]), radioactive wastes, etc. The reservoir is usually a multi-layered domain composed by some superposed aquifers separated by less permeable layers. In addition, fractured zones can divide the domain in blocks which can slide between each other. The steady one phase flow equation in saturated porous media is derived from the mass conservation law and the linear Darcy's law and can be written (in its simplest form) as:

$$
-\operatorname{div}(k \nabla u)=f
$$

where the unknown $u$ is the pressure, $k$ is the permeability and $f$ denotes a possible sink/source term. Obviously, the flow problem is obtained by adding some boundary conditions. For the multi-layered porous media, each layer is assumed homogeneous and the permeability is a piecewise constant function. Therefore, the interfaces between the layers represent the discontinuities of the permeability, but the intrinsic variables (pressure and flux) are continuous in all the domain, notably on interfaces. 


\section{Non-overlapping DDMs}

From the DD point of view, the geological layers induce a natural nonoverlapping decomposition. The DD being already set, we must define appropriate algorithms to obtain efficient DDMs. For simplicity of the description, we consider only two layers, i.e. the domain $\Omega$ is decomposed in two subdomains $\Omega_{1}, \Omega_{2}$, with $\Gamma$ the common interface and $k_{i}$ the permeability of layer $i$. The steady flow problem can be written as a transmission problem:

$$
\begin{gathered}
-k_{1} \Delta u_{1}=f \text { in } \Omega_{1} \quad, \quad-k_{2} \Delta u_{2}=f \text { in } \Omega_{2} \\
\left.u_{1}\right|_{\Gamma}=\left.u_{2}\right|_{\Gamma} \quad, \quad k_{1} \partial u_{1} /\left.\partial n_{1}\right|_{\Gamma}=-k_{2} \partial u_{2} /\left.\partial n_{2}\right|_{\Gamma}
\end{gathered}
$$

with appropriate boundary conditions on $\partial \Omega$.

The multiplicative D-N algorithm requires to solve successively $(n \geq 0)$ :

$$
\left\{\begin{array} { l } 
{ - k _ { 1 } \Delta u _ { 1 } ^ { n + 1 } = f \text { in } \Omega _ { 1 } } \\
{ u _ { 1 } ^ { n + 1 } | _ { \Gamma } = u _ { 2 } ^ { n } | _ { \Gamma } }
\end{array} \quad \left\{\begin{array}{l}
-k_{2} \Delta u_{2}^{n+1}=f \text { in } \Omega_{2} \\
-k_{2} \partial u_{2}^{n+1} /\left.\partial n_{2}\right|_{\Gamma}=k_{1} \partial u_{1}^{n+1} /\left.\partial n_{1}\right|_{\Gamma}
\end{array}\right.\right.
$$

Since it uses both physical interface conditions (3), this algorithm seems the simplest and the most adapted to the physics of the problem.

An alternative to the D-N algorithm is the Robin-Method in which weighted sums of physical conditions are used:

$$
\begin{array}{ll}
\left.\alpha_{1} u_{1}\right|_{\Gamma}+\beta_{1} k_{1} \partial u_{1} /\left.\partial n_{1}\right|_{\Gamma}=\left.\alpha_{1} u_{2}\right|_{\Gamma}-\beta_{1} k_{2} \partial u_{2} /\left.\partial n_{2}\right|_{\Gamma} \\
\left.\alpha_{2} u_{2}\right|_{\Gamma}+\beta_{2} k_{2} \partial u_{2} /\left.\partial n_{2}\right|_{\Gamma}=\left.\alpha_{2} u_{1}\right|_{\Gamma}-\beta_{2} k_{1} \partial u_{1} /\left.\partial n_{1}\right|_{\Gamma}
\end{array}
$$

The use of RC for non-overlapping DD has been firstly proposed by P.-L. Lions (Proc. DDM3, 202-223, 1990). It is easy to prove that, if $\alpha_{1} \beta_{2}+\alpha_{2} \beta_{1} \neq 0$, then conditions (3) and (4) are algebraically equivalent. In addition, conditions $\alpha_{i} \beta_{i} \geq 0$ have to be verified to obtain well-posed sub-problems. Since conditions (3) can be obtained from (4) by considering $\alpha_{1}=\beta_{2}=1, \alpha_{2}=\beta_{1}=0$, the D-N algorithm could be seen like a particular case of Robin-Method.

Both algorithms present the same inconvenience: the convergence is not ensured. Indeed, as shown in the next sections, the convergence of the DirichletNeumann algorithm depends on the interface conditions distribution between the domains, while the convergence of the second algorithm depends on the choice of the Robin's coefficients $\alpha_{i}, \beta_{i}$.

\section{Improved non-overlapping DDMs}

To cure such an inconvenience, several methods have been already proposed in DD literature (with or without overlap) for linear elliptic problems.

A recent method is an acceleration of Aitken type of the iterative solutions obtained by Schwarz algorithm and restricted to the interfaces. This method 
has been introduced by Garbey and Tromeur-Dervout (Proc. DDM12, 325339,2000 ), and studied theoretically and numerically by Garbey and TromeurDervout [2002] for the additive Schwarz algorithm and 1-D decompositions. Some numerical experiences have also been described for the D-N algorithm. The basic idea of the Aitken technique is to accelerate independently each mode of the sine expansion of the iterative solutions restricted to the interfaces. In these papers, the method is developed at semi-discrete level (the problem is uniformly discretized in the interface direction). Some developments have been proposed to generalize Aitken acceleration for irregular grids (Baranger et al., Proc. DDM13, 287-294, 2001) or for non-matching grids (Baranger et al., Proc. DDM14, 341-348, 2002). The case of 2-D decompositions is treated considering some 1-D decompositions in a recursive manner (Garbey and Tromeur-Dervout, Proc. DDM13, 53-65, 2001) or accelerating the signals obtained by representing the discrete interface solutions in Fourier spaces (Calugaru and Tromeur-Dervout, Proc. Parallel CFD 2003, to appear).

A second method is to find $\mathrm{RC}$ which allow a fast convergence. This idea has been introduced by Després et al. [1992] for Helmholtz and Maxwell problems, and by Nataf et al. [1994] for convection-diffusion equations. It has been also used for flow problems in heterogeneous porous media (Faille et al. [2001]). In general, one can suppose $\beta_{i}=1$ and then only $\alpha_{i}$ coefficients are searched. Optimized Robin conditions (ORC) could be also introduced as the best zeroth order approximations of optimal interface conditions (see for instance, Gander et al. [2002] for Helmholtz equation). In this context, it is possible to define other interface conditions (as for example, second order approximations), but which are not investigated in this paper.

A third method (the first in chronological order) was proposed by Funaro, Quarteroni and Zanolli (SIAM J. Num. Anal., 25(6), 1213-1236, 1988) and consists of modifying the multiplicative D-N algorithm by using a relaxation procedure at the end of each iteration. If the relaxation parameter is conveniently chosen, then the convergence is obtained.

In the next sections, for flow problems in multi-layered porous media, we investigate only two multiplicative algorithms: the D-N algorithm accelerated by an Aitken technique (A-D-N) and the Robin-Method. The convergence of these methods is studied in 1-D and 2-D frameworks and a numerical comparison is presented.

\section{Convergence in 1-D framework}

Let us consider the problem (2)-(3) for $\Omega=(a, b)$, with Dirichlet boundary condition $u_{D}$ and $\Gamma=\{\lambda\} \subset(a, b)$ the common interface.

The (multiplicative) Robin-Method reads: 


$$
\begin{aligned}
& \left\{\begin{array}{l}
-k_{1}\left(u_{1}^{n+1}\right)^{\prime \prime}=f \text { in }(a, \lambda) \\
u_{1}^{n+1}(a)=u_{D}(a) \\
\alpha_{1} u_{1}^{n+1}(\lambda)+\beta_{1} k_{1}\left(u_{1}^{n+1}\right)^{\prime}(\lambda)=\alpha_{1} u_{2}^{n}(\lambda)+\beta_{1} k_{2}\left(u_{2}^{n}\right)^{\prime}(\lambda)
\end{array}\right. \\
& \left\{\begin{array}{l}
-k_{2}\left(u_{2}^{n+1}\right)^{\prime \prime}=f \text { in }(\lambda, b) \\
u_{2}^{n+1}(b)=u_{D}(b) \\
\alpha_{2} u_{2}^{n+1}(\lambda)-\beta_{2} k_{2}\left(u_{2}^{n+1}\right)^{\prime}(\lambda)=\alpha_{2} u_{1}^{n+1}(\lambda)-\beta_{2} k_{1}\left(u_{1}^{n+1}\right)^{\prime}(\lambda)
\end{array}\right.
\end{aligned}
$$

where $u_{2}^{0}(\lambda),\left(u_{2}^{0}\right)^{\prime}(\lambda)$ are arbitrarily chosen.

To analyze the convergence of this algorithm, it suffices by linearity to consider homogeneous problem $\left(f \equiv 0, u_{D} \equiv 0\right)$ and to analyze convergence to zero. Solving successively the above ODEs and denoting $d_{1}=\lambda-a, d_{2}=b-\lambda$, we obtain $u_{1}^{n+2}(\lambda)=\rho\left(\alpha_{1}, \beta_{1}, \alpha_{2}, \beta_{2}\right) u_{1}^{n}(\lambda)$, with the convergence rate:

$$
\rho\left(\alpha_{1}, \beta_{1}, \alpha_{2}, \beta_{2}\right)=\frac{\alpha_{1} d_{2}-\beta_{1} k_{2}}{\alpha_{1} d_{1}+\beta_{1} k_{1}} \cdot \frac{\alpha_{2} d_{1}-\beta_{2} k_{1}}{\alpha_{2} d_{2}+\beta_{2} k_{2}}
$$

\subsection{Aitken-Dirichlet-Neumann algorithm}

For he D-N algorithm the convergence rate becomes: $\rho_{D N}=-d_{2} / d_{1} \cdot k_{1} / k_{2}$. Therefore, the convergence of the D-N algorithm is determined only by the ratios of subdomains lengths and of permeabilities. These values being fixed, if the algorithm diverges, it is not possible to adjust any parameter to achieve convergence. The only one possibility is the inter-changing of the interface conditions, but this technique is not easy to handle in practice, where complex basins presenting many porous blocks with extreme contrasts in permeability have to be taken into account. Moreover, in some situations the inter-changing of the interface conditions may lead to an ill-posed problem (only Neumann conditions on all boundaries of a subdomain), which is the typical "danger" of the D-N algorithm.

To transform the D-N algorithm into an attractive algorithm, we use the Aitken acceleration of the traces of the iterative solutions on the common interface. This method is based on the linear behavior of the error at interfaces for the D-N algorithm applied to a linear elliptic operator, as it is here. Indeed, as shown by Garbey and Tromeur-Dervout [2002] in linear cases, the error of the multiplicative $\mathrm{D}-\mathrm{N}$ algorithm satisfies:

$$
u_{2}^{n+1}(\lambda)-u_{2}^{\infty}(\lambda)=\delta\left(u_{2}^{n}(\lambda)-u_{2}^{\infty}(\lambda)\right) \text { for all } n \in \mathbb{N}
$$

The first step of the Aitken technique is to compute the damping factor $\delta$. For the considered problem, its value is already known (it is exactly $\rho_{D N}$ ). Then, we can pass directly to the Aitken acceleration step, which gives the exact value $u_{2}^{\infty}(\lambda)$ from $(6)$ as follows: $u_{2}^{\infty}(\lambda)=\left(u_{2}^{1}(\lambda)-\delta u_{2}^{0}(\lambda)\right) /(1-\delta)$, after one D-N iteration. An additional iteration suffices to obtain solution in all domain. In conclusion, for the considered problem, we need only two iterations 
of D-N algorithm. For other problems, the second step remains non-changed, but only the first step (computation of $\delta$ ) is modified. This can be done in analytical way, when the operator is still relatively simple. Elsewhere, one can compute the damping factor $\delta$ numerically, by performing two iterations of $\mathrm{D}-\mathrm{N}$ algorithm (and using the obtained iterative solutions on the interface in (6), for $n=0,1)$. Therefore, in the general linear case, we need three iterations of D-N algorithm to obtain the exact solutions in all domain.

\subsection{Optimized Robin-Method}

In 1-D framework, the coefficients that minimize the convergence rate are obtained immediately from (5). Indeed, considering $\beta_{1}=\beta_{2}=1$, we obtain $\rho_{O R C}=0$ for the following optimal coefficients: $\alpha_{1, \text { opt }}=k_{2} / d_{2}, \alpha_{2, \text { opt }}=k_{1} / d_{1}$. Consequently, after only one iteration one obtains exact interface values. As for the A-D-N algorithm, an additional iteration (using exact interface values) allows the complete computation of the solution. Then, for the considered problem, this method requires only two iterations.

If we have a different problem to solve, the convergence rate expression must be analytically deduced and one obtains a relation similar to (5). For usual operators, optimal coefficients can be directly deduced from such an expression in order to obtain a null convergence rate. Then, the exact solution is still obtained after only two iterations. However, a more complicated operator can lead to a more complicated expression of the convergence rate, and it is possible to be not able to deduce analytically the optimal coefficients or/and the optimal rate is not zero. In this case, a numerical optimization procedure can be used or/and the method is not exact, but iterative.

\section{Convergence in 2-D framework}

Consider now the problem (2)-(3) in 2-D framework. Firstly, we consider an infinite domain $\Omega=\mathbb{R}^{2}$, with $\Omega_{1}=(-\infty, 0) \times \mathbb{R}, \Omega_{2}=(0, \infty) \times \mathbb{R}$ and suppose that the solution is bounded. Using the Fourier transform in the $y$ direction (with $\xi$ the frequency variable), the Robin-Method yields in Fourier space:

$$
\begin{aligned}
& \left\{\begin{array}{l}
-k_{1}\left[\left(\widehat{u}_{1}^{n+1}\right)_{x x}(x, \xi)-\xi^{2} \widehat{u}_{1}^{n+1}(x, \xi)\right]=\widehat{f}(x, \xi), \text { in }(-\infty, 0) \times \mathbb{R} \\
\alpha_{1} \widehat{u}_{1}^{n+1}(0, \xi)+\beta_{1} k_{1}\left(\widehat{u}_{1}^{n+1}\right)_{x}(0, \xi)=\alpha_{1} \widehat{u}_{2}^{n}(0, \xi)+\beta_{1} k_{2}\left(\widehat{u}_{2}^{n}\right)_{x}(0, \xi), \xi \in \mathbb{R}
\end{array}\right. \\
& \left\{\begin{array}{l}
-k_{2}\left[\left(\widehat{u}_{2}^{n+1}\right)_{x x}(x, \xi)-\xi^{2} \widehat{u}_{2}^{n+1}(x, \xi)\right]=\widehat{f}(x, \xi), \text { in }(0, \infty) \times \mathbb{R} \\
\alpha_{2} \widehat{u}_{2}^{n+1}(0, \xi)-\beta_{2} k_{2}\left(\widehat{u}_{2}^{n+1}\right)_{x}(0, \xi)=\alpha_{2} \widehat{u}_{1}^{n+1}(0, \xi)-\beta_{2} k_{1}\left(\widehat{u}_{1}^{n+1}\right)_{x}(0, \xi), \xi \in \mathbb{R}
\end{array}\right.
\end{aligned}
$$

As in 1-D framework, solving successively the above ODEs with boundedness conditions for $\widehat{u}_{1}^{n+1}, \widehat{u}_{2}^{n+1}$, we obtain the convergence rate:

$$
\rho\left(\alpha_{1}, \beta_{1}, \alpha_{2}, \beta_{2}, \xi\right)=\frac{\alpha_{1}-\beta_{1} k_{2}|\xi|}{\alpha_{1}+\beta_{1} k_{1}|\xi|} \cdot \frac{\alpha_{2}-\beta_{2} k_{1}|\xi|}{\alpha_{2}+\beta_{2} k_{2}|\xi|}, \quad \forall \xi \in \mathbb{R}
$$




\subsection{Aitken-Dirichlet-Neumann algorithm}

For the D-N algorithm, the convergence rate becomes: $\rho_{D N}(\xi)=-k_{1} / k_{2}$, for all $\xi \in \mathbb{R}$, i.e. all frequency components have the same convergence rate. Then, the algorithm converges or diverges according to the distribution of interface conditions in the two subdomains. This convergence rate being also valid in the physical space, the Aitken acceleration can be applied directly to the iterative solutions in each point within the subdomains.

However, the results obtained in analysis of unbounded cases can be not relevant for the bounded case. We can illustrate this situation, considering the domain is bounded only in $x$ direction, as for instance, $\Omega=(0,1) \times \mathbb{R}$, with common interface $\Gamma=\{\lambda\} \times \mathbb{R}, 0<\lambda<1$. On the lateral boundaries, homogeneous Dirichlet conditions are imposed. Using the same Fourier analysis as above, one obtains the convergence rate:

$$
\rho_{D N}(\xi)=\frac{k_{1}}{k_{2}} \cdot \frac{1-e^{2|\xi|}}{1+e^{2|\xi|}} \cdot \frac{1+e^{-\lambda|\xi|}}{1-e^{-\lambda|\xi|}}, \quad \forall \xi \in \mathbb{R}
$$

In this semi-bounded case, each frequency component has its own own linear damping factor. The Aitken acceleration is no longer possible in the physical space, but can be applied for each frequency component by using the numerical 1-D procedure described in $\S 4.1$.

For realistic domains (bounded in both directions), we use the Aitken technique as follows: let $\left\{P_{i}\right\}_{i=1, \ldots, N}$ the discrete representation of the common interface $\Gamma$ (we consider a regular discretization in $\Gamma$ direction). When D-N algorithm is applied, at discrete level we obtain the traces of iterative discrete solutions, denoted $\left\{u_{2, i}^{n}\right\}_{i=1, \ldots, N}$ which are transformed in periodic signals, and then represented in mode's Fourier space. Then, the Aitken acceleration is possible for each mode, since each mode is damped linearly. Using accelerated modes, the solution is recomposed in the physical space.

\subsection{Optimized Robin-Method}

Let $\beta_{1}=\beta_{2}=1$ in (7). Applying the technique introduced by Nataf and coauthors in papers cited above, for the considered problem, we obtain optimized Robin coefficients $\alpha_{1, \text { opt }}, \alpha_{2, \text { opt }}$ by solving the min-max problem:

$$
\min _{\alpha_{1}, \alpha_{2}>0}\left(\max _{\xi \in \mathbb{R}}\left|\frac{\alpha_{1}-k_{2}|\xi|}{\alpha_{1}+k_{1}|\xi|} \cdot \frac{\alpha_{2}-k_{1}|\xi|}{\alpha_{2}+k_{2}|\xi|}\right|\right)
$$

Since real computations are performed on bounded domains and discretized operators, the range of $\xi$ can be bounded in an interval $\left(\xi_{\min }, \xi_{\max }\right)$. Even with this simplification, the problem (9) is still difficult to solve analytically. The method given by Faille et al. [2001] divides the previous problem into two auxiliary min-max problems for $\alpha_{1}$ and respectively for $\alpha_{2}$, which are 
formulated as for homogeneous media. The problems are similar to optimal parameter search in ADI Peaceman-Racheford method and have the solutions:

$$
\alpha_{1, \text { opt }}=k_{2} \sqrt{\xi_{\min } \xi_{\max }} \quad, \quad \alpha_{2, \text { opt }}=k_{1} \sqrt{\xi_{\min } \xi_{\max }}
$$

Another method retained here is to solve (9) at discrete level, for the heterogeneous case, by considering $\alpha_{1}=\alpha_{2} \equiv \alpha$. For instance, we replaced $L^{\infty}-$ norm by the discrete $L^{1}$-norm of frequency components in $\left(\xi_{\min }, \xi_{\max }\right)$ and then, the $\alpha_{o p t, L^{1}}$ is obtained by seeking the minimum for a fine mesh for $\alpha$.

\section{Numerical results}

We consider the problem (2)-(3) for $\Omega_{1}=(0, \pi)^{2}, \Omega_{2}=(\pi, 2 \pi) \times(0, \pi)$, $f(x, y)=2 k_{1} k_{2} \sin x \sin y$ and the Dirichlet condition $u=10$. The exact solution is $u_{1}(x, y)=10+k_{2} \sin x \sin y, u_{2}(x, y)=10+k_{1} \sin x \sin y$. We consider $k_{1}=10, k_{2}=1$, and $u_{2}^{0}(\pi, y)=\left(u_{2}^{0}\right)_{x}(\pi, y)=0$, for $y \in(0, \pi)$.

Figure 1 shows the evolution of the error with respect to the iterations for the investigated algorithms. One can observe that even if the D-N algorithm diverges rapidly, the Aitken acceleration (A-D-N on the Figure 1) allows a fast convergence of the algorithm.

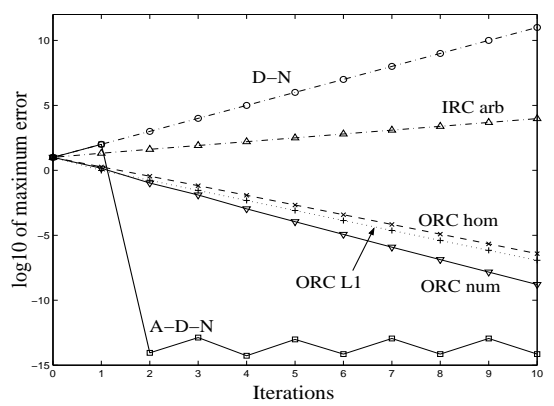

Figure 1.

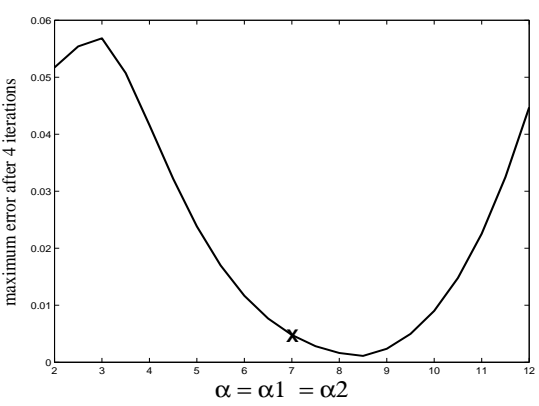

Figure 2.

The Robin-Method can diverge if the RC are chosen arbitrarily, as for instance, using $\alpha_{i}=k_{i}$ (curve IRCarb). Now, let us consider optimized RC. Two optimized Robin-Methods have been investigated: the ORChom method which gives $\alpha_{1, o p t}=3, \alpha_{2, o p t}=30$ from (10) and the ORCL1 method which gives $\alpha_{o p t, L^{1}} \simeq 7$. For the two methods, the obtained results are relatively close: in 10 iterations the error is reduced by a factor of $10^{7}$.

Both ORC are obtained by solving (9). However, this problem being deduced with a Fourier analysis at continuous level for unbounded domain, the obtained ORC are not necessarily optimal at the discrete level for the bounded domain used in the numerical experiment. To verify how the coefficients obtained with ORCL1 method approach the optimal discrete coefficients, Figure 
2 shows the error reduction obtained numerically, after 4 iterations, using various values for $\alpha=\alpha_{1}=\alpha_{2}$. We observe that the coefficient obtained by the optimization procedure $(\sim 7)$, is not very close of the discrete optimum, but it can give values which are effective for numerical experiments. Using the discrete optimum ( 8.5), the obtained error after 10 iterations (curve ORCnum) is better with a factor $10^{2}$ with respect to the ORCL1 method.

\section{Conclusions}

We studied two non-overlapping methods for flow problem in heterogeneous porous media: A-D-N method and Optimized Robin-Method. Both methods use the Fourier analysis but at different level. In the A-D-N method, the discrete solution is represented in modes's space, accelerated and transformed back in the physical space. For the second method, the Fourier analysis is used only to determine optimized Robin coefficients. Both methods show good convergence properties, especially the Aitken method. However, there are several possibilities to improve the Optimized Robin-Method, as the use of a Krylov acceleration, or the use of second order optimized interface conditions (OIC2). It is also possible to apply Aitken acceleration to the Robin-Method (not necessarily optimized), because it is still linear. The two methods, although studied here for only 2 subdomains have been already used to an arbitrary number of subdomains, in 1-D or 2-D framework (Calugaru and TromeurDervout, Proc. Parallel CFD 2003, to appear, for the A-D-N method and Faille et al. [2001] for the Robin-Method). We are currently investigating a comparison of these extensions in the context of parallelization methods.

\section{References}

D.-G. Calugaru, J.-M. Crolet, A. Chambaudet, and F. Jacob. Radon transport as an indicator of seismic activity. An algorithm for inverse problems. Computational Methods in Water Ressources, 47:631-638, 2002.

B. Després, P. Joly, and J. E. Roberts. A domain decomposition method for the harmonic Maxwell equations. In Iterative methods in linear algebra (Brussels, 1991), pages 475-484, Amsterdam, 1992. North-Holland.

I. Faille, E. Flauraud, F. Nataf, F. Schneider, and F. Willien. Optimized interface conditions for sedimentary basin modeling. In N. Debit et al., editor, Proc. of the 13th Int. Conf. on DDM, pages 461-468, 2001.

M. J. Gander, F. Magoulès, and F. Nataf. Optimized Schwarz methods without overlap for the Helmholtz eq. SIAM J. Sci. Comput., 24(1):38-60, 2002.

M. Garbey and D. Tromeur-Dervout. On some Aitken acceleration of the Schwarz method. Int. J. for Num. Meth. in Fluids, 40(12):1493-1513, 2002.

F. Nataf, F. Rogier, and E. de Sturler. Optimal interface conditions for domain decomposition methods. Technical Report 301, CMAP, 1994. 